\title{
The Efficiency Of OLS In The Presence Of Auto- Correlated Disturbances In Regression Models
}

\author{
Samir Safi \\ James Madison University \\ Alexander White \\ Texas State University
}

Follow this and additional works at: http://digitalcommons.wayne.edu/jmasm

Part of the Applied Statistics Commons, Social and Behavioral Sciences Commons, and the Statistical Theory Commons

\section{Recommended Citation}

Safi, Samir and White, Alexander (2006) "The Efficiency Of OLS In The Presence Of Auto-Correlated Disturbances In Regression Models," Journal of Modern Applied Statistical Methods: Vol. 5 : Iss. 1 , Article 10.

DOI: $10.22237 /$ jmasm/1146456540

Available at: http://digitalcommons.wayne.edu/jmasm/vol5/iss1/10 


\section{The Efficiency Of OLS In The Presence Of Auto-Correlated Disturbances In Regression Models}

\author{
Samir Safi \\ Department of Mathematics and Statistics \\ James Madison University*
}

\author{
Alexander White \\ Department of Mathematics and Statistics \\ Texas State University
}

The ordinary least squares (OLS) estimates in the regression model are efficient when the disturbances have mean zero, constant variance, and are uncorrelated. In problems concerning time series, it is often the case that the disturbances are correlated. Using computer simulations, the robustness of various estimators are considered, including estimated generalized least squares. It was found that if the disturbance structure is autoregressive and the dependent variable is nonstochastic and linear or quadratic, the OLS performs nearly as well as its competitors. For other forms of the dependent variable, rules of thumb are presented to guide practitioners in the choice of estimators.

Key words: Autocorrelation, autoregressive, ordinary least squares, generalized least squares, efficiency

\section{Introduction}

Let the relationship between an observable random variable $\mathrm{y}$ and $\mathrm{k}$ explanatory variables $\mathrm{X}_{1}, \mathrm{X}_{2}, \ldots, \mathrm{X}_{\mathrm{k}}$ in a $\mathrm{T}$-finite system be specified in the following linear regression model:

$$
\mathrm{y}=\mathrm{X} \beta+\mathrm{u}
$$

where $y$ is a $(T \times 1)$ vector of observations on a response variable, $\mathrm{X}$ is a $(\mathrm{T} \times \mathrm{k})$ design matrix, $\beta$ is a $(\mathrm{k} \times 1)$ vector of unknown regression parameters, and $\mathrm{u}$ is a $(\mathrm{T} \times 1)$ random vector of disturbances. For convenience, it is assumed that

*This article was accepted while Samir Safi was at James Madison University. $\mathrm{He}$ is now an Assistant Professor of Statistics at the Islamic University of Gaza. His research interests are in time series analysis, concerning the comparison of estimators in regression models with autocorrelated disturbances and efficiency of OLS in the presence of autocorrelated disturbances. Alexander White is Associate Professor in Mathematics Education. His research interests are in mathematics education, statistics and mathematical finance.
$\mathrm{X}$ is full column rank $\mathrm{k}<\mathrm{T}$ and its first column is 1's. The ordinary least squares (OLS) estimator of $\beta$ in the regression model (1) is

$$
\hat{\beta}=\left(X^{\prime} X\right)^{-1} X^{\prime} y
$$

In problems concerning time series, it is often the case that the disturbances are, in fact, correlated. Practitioners are then faced with a decision, use OLS anyway, or try to fit a more complicated disturbance structure. The problem is difficult because the properties of the estimators depend highly on the structure of the independent variables in the model. For more complicated disturbance structures, many of the properties are not well understood. If the disturbance term has mean zero, i.e. $\mathrm{E}(\mathrm{u})=0$, but is in fact, autocorrelated, i.e. $\operatorname{Cov}(\mathrm{u})=\sigma_{\mathrm{u}}^{2} \Sigma$, where $\Sigma$ is a $\mathrm{T} \times \mathrm{T}$ positive definite matrix and the variance $\sigma_{u}^{2}$ is either known or unknown positive and finite scalar, then the OLS parameter estimates will continue to be unbiased, i.e. $\mathrm{E}(\hat{\beta})=\beta$. But it has a different covariance matrix;

$$
\operatorname{Cov}_{\Sigma}(\hat{\beta})=\sigma_{u}^{2}\left(X^{\prime} X\right)^{-1} X^{\prime} \sum X\left(X^{\prime} X\right)^{-1}
$$


The most serious implication of autocorrelated disturbances is not the resulting inefficiency of OLS, but the misleading inference when standard tests are used. The autocorrelated nature of disturbances is accounted for in the generalized least squares (GLS) estimator given by:

$$
\widetilde{\beta}=\left(X^{\prime} \Sigma^{-1} X\right)^{-1} X^{\prime} \Sigma^{-1} y
$$

which is unbiased, i.e. $\mathrm{E}(\widetilde{\beta})=\beta$, with covariance matrix

$$
\operatorname{Cov}(\widetilde{\beta})=\sigma_{u}^{2}\left(X^{\prime} \Sigma^{-1} X\right)^{-1}
$$

The superiority of GLS over OLS is due to the fact that GLS has a smaller variance. According to the Generalized Gauss Markov Theorem, the GLS estimator provides the Best Linear Unbiased Estimator (BLUE) of $\beta$. But the GLS estimator requires prior knowledge of the matrix correlation structure, $\Sigma$. The OLS estimator $\hat{\beta}$ is simpler from a computational point of view and does not require a prior knowledge of $\Sigma$.

A common approach for modeling univariate time series is the autoregressive model. The general finite order autoregressive process of order $p$ or briefly, $\mathrm{AR}(\mathrm{p})$, is

$$
\begin{aligned}
& \mathrm{u}_{\mathrm{t}}=\phi_{1} \mathrm{u}_{\mathrm{t}-1}+\phi_{2} \mathrm{u}_{\mathrm{t}-2}+\cdots+\phi_{\mathrm{p}} \mathrm{u}_{\mathrm{t}-\mathrm{p}}+\varepsilon_{\mathrm{t}}, \varepsilon_{\mathrm{t}} \sim \\
& \text { i.i.d. } \mathrm{N}\left(0, \sigma_{\varepsilon}^{2}\right)
\end{aligned}
$$

There are numerous articles describing the efficiency of the OLS coefficient estimator $\hat{\beta}$, which ignore the correlation of the error, relative to the GLS estimator $\widetilde{\beta}$, which takes this correlation into account. One strand is concerned with conditions on regressors and error correlation structure, which guarantee that OLS is asymptotically as efficient as GLS (e.g. Chipman, 1979; Krämer, 1980). The efficiency of the OLS estimators in a linear regression containing an autocorrelated error term depends on the structure of the matrix of observations on the independent variables (e.g. Anderson, 1948; 1971; Grenander \& Rosenblatt, 1957).
For a linear regression model with first order autocorrelated disturbances, several alternative estimators for the regression coefficients have been discussed in the literature, and their efficiency properties have been investigated with respect to the OLS and GLS estimators (e.g. Kadiyala, 1968; Maeshiro, 1976; 1979; Ullah et al., 1983).

The relative efficiency of GLS to OLS in the important cases of autoregressive disturbances of order one, $\operatorname{AR}(1)$, with autoregressive coefficient $\rho$ and second order, $\operatorname{AR}(2)$, with autoregressive coefficients $\left(\phi_{1}, \phi_{2}\right)$ for specific choices of the design vector have been investigated.

Building on work on the economics and time series literature, the price one must pay for using OLS under suboptimal conditions required investigation. Different designs are being explored, under which relative efficiency of the OLS estimator to that of GLS estimator approaches to one or zero, determining ranges of first-order autoregressive coefficient, $\rho$, in $\mathrm{AR}(1)$ disturbance and second order of autoregressive coefficients, $\left(\phi_{1}, \phi_{2}\right)$ in $\operatorname{AR}(2)$ for which OLS is efficient and quantifying the effect of the design on the efficiency of the OLS estimator. Furthermore, a simulation study has been conducted to examine the sensitivity of estimators to model misspecification. In particular, how do estimators perform when an $\mathrm{AR}(2)$ process is appropriate and the process is incorrectly assumed to be an $\mathrm{AR}(1)$ or $\mathrm{AR}(4)$ ?

\section{Performance Comparisons}

In this section, numerical results are presented using the formulas in (3) and (5). Focus will be placed on two issues; first, the relative efficiency of GLS estimator as compared with the OLS estimator when the structure of the design vector, $X$, is nonstochastic. For example, linear, quadratic, and exponential design vectors with an intercept term included in the design vector. Secondly, the relative efficiency of the GLS estimator as compared with the OLS for a stochastic design vector. In the example considered here, a standard Normal stochastic design vector of length 1000 was generated. The three finite sample sizes used are 50, 100, and 200 for 
selected values of the autoregressive coefficients. Both $\mathrm{AR}(1)$ and $\mathrm{AR}(2)$ error processes are considered to discuss the behavior of OLS as compared to GLS.

Performance Comparisons for AR (1) Process

The relative efficiencies of OLS to GLS are discussed when the disturbance term follows an $\operatorname{AR}(1)$ process, $u_{t}=\rho u_{t-1}+\varepsilon_{t}, t=1,2, \ldots, T$, assuming that the autoregressive coefficient, $\rho$, is known priori. The three finite sample sizes used are 50, 100, and 200 for the elected values of $|\rho| \leq .9$, evaluated in steps of .2 .

Table (1) shows the relative efficiencies of the variances of GLS to OLS for a regression coefficient on linear trend with an intercept term included in the design. For estimating an intercept term, the relative efficiency of the OLS estimator as compared to the GLS estimator decreases with increasing values of $|\rho|$. For small and moderate sample sizes, the efficiency of the OLS estimator appears to be nearly as efficient as the GLS estimator for $|\rho| \leq .7$. In addition, for large size sample data, the OLS estimator performs nearly as efficiently as the GLS estimator for the additional values of $\rho= \pm$.9. Further, the efficiency for estimating the slope mimics the efficiency of the intercept, except for large sample size; the efficiency of the OLS estimator appears to be nearly as efficient as the GLS estimator for $\rho \neq \pm$.9.

The efficiency of GLS estimator to the OLS estimator for the quadratic design agrees with the behavior for the linear design vector. In contrast, the gain in efficiency of the GLS estimator for different design vectors such as exponential and 1000 standard Normal, $\mathrm{N}(0,1)$

Table 1: Relative Efficiency of GLS to OLS for Linear Design

\begin{tabular}{ccccccc}
\hline$\rho$ & & Intercept & & & Slope & \\
\cline { 2 - 6 } & $\mathrm{T}=50$ & $\mathrm{~T}=100$ & $\mathrm{~T}=200$ & $\mathrm{~T}=50$ & $\mathrm{~T}=100$ & $\mathrm{~T}=200$ \\
\hline-0.9 & 0.7097 & 0.8276 & 0.9047 & 0.6739 & 0.8012 & 0.8881 \\
-0.7 & 0.9162 & 0.9552 & 0.9768 & 0.9024 & 0.9471 & 0.9724 \\
-0.5 & 0.9694 & 0.9840 & 0.9918 & 0.9640 & 0.9810 & 0.9903 \\
-0.3 & 0.9908 & 0.9952 & 0.9976 & 0.9891 & 0.9943 & 0.9971 \\
-0.1 & 0.9991 & 0.9995 & 0.9998 & 0.9989 & 0.9994 & 0.9997 \\
0.1 & 0.9991 & 0.9995 & 0.9998 & 0.9989 & 0.9994 & 0.9997 \\
0.3 & 0.9911 & 0.9953 & 0.9976 & 0.9894 & 0.9944 & 0.9971 \\
0.5 & 0.9717 & 0.9846 & 0.9920 & 0.9662 & 0.9816 & 0.9904 \\
0.7 & 0.9288 & 0.9585 & 0.9777 & 0.9147 & 0.9503 & 0.9732 \\
0.9 & 0.8359 & 0.8691 & 0.9164 & 0.8000 & 0.8418 & 0.8993
\end{tabular}


compared to the OLS estimator is substantial for moderate and large values of $\rho$. However, for small values of $\rho$ the OLS appears to be nearly as efficient as GLS.

\section{Performance Comparisons for AR (2) Process}

The relative efficiencies of OLS to GLS are discussed for linear, quadratic, and exponential design vectors when the disturbance term follows an $\operatorname{AR}(2)$ process, $\mathrm{u}_{\mathrm{t}}=\phi_{1} \mathrm{u}_{\mathrm{t}-1}+\phi_{2} \mathrm{u}_{\mathrm{t}-2}+\varepsilon_{\mathrm{t}}, \mathrm{t}=1,2, \ldots, \mathrm{T}, \quad$ assuming that the autoregressive coefficients $\phi_{1}$ and $\phi_{2}$ are known priori. The three finite sample sizes used are 50, 100, and 200 for the selected 45 pairs of the autoregressive coefficients. These coefficients were chosen according to stationary conditions $\left(\phi_{1}+\phi_{2}<1, \phi_{2}-\phi_{1}<1\right.$, and $\left.\left|\phi_{2}\right|<1\right)$ and so that $\rho_{1}=\phi_{1}\left(1-\phi_{2}\right)^{-1}$ is positive. This second condition was chosen since this is the case in most econometric studies.

To demonstrate the efficiency of OLS, consider the linear design vector. When the disturbance term follows an $\mathrm{AR}(2)$ process for the linear design with small sample size, OLS performs nearly as efficiently as GLS for estimating the slope for all $\operatorname{AR}(2)$ parametrizations except when $\phi$ 's are close to the stationary boundary. As the sample size increases, the difference between the performance of OLS and GLS decreases. Only when $\phi_{2}=-.9$, does OLS perform badly regardless of the sample size. The efficiency of GLS to OLS for the quadratic design mimics the behavior for the linear design. Finally, for exponential and 1000 standard Normal design vectors, the efficiency of OLS appears to be nearly as efficient as GLS for $\phi_{1}=.2$ and small values of $\phi_{2} 2$ for all sample sizes. Otherwise, OLS performs poorly.

\section{Simulation Study}

In this section, the robustness of various estimators are considered, including estimated generalized least squares (EGLS). These simulations examine the sensitivity of estimators to model misspecification. In particular, how do estimators perform when an $\mathrm{AR}(2)$ process is appropriate and it is incorrectly assumed that the process is an $\mathrm{AR}(1)$ ? The finite sample efficiencies of the OLS estimator relative to four GLS estimators are compared: the GLS based on the correct disturbance model structures and known AR(2) coefficients denoted as GLS$\operatorname{AR}(2)$; the GLS based on the correct disturbance model structures, but with estimated AR(2) coefficients denoted as EGLS-AR(2); the GLS based on $\mathrm{AR}(1)$ incorrect disturbance model structures with an estimated AR(1) coefficient denoted as EIGLS-AR(1); and the GLS based on $\mathrm{AR}(4)$ incorrect disturbance model structures with estimated $\mathrm{AR}(4)$ coefficients denoted as EIGLS-AR(4). This study focuses only on $\mathrm{AR}(\mathrm{p})$ GLS corrections disturbances which are widely used in econometric studies.

\section{The Simulation Setup}

Three finite sample sizes $(50,100$, and 200) and three nonstochastic design vectors of the independent variable are used; linear, quadratic, and exponential. A standard Normal stochastic design vector of length 1000 is also generated (Assuming that the variance of the error term in $\operatorname{AR}(2)$ process $\left.\sigma_{\varepsilon}^{2}=1\right)$. Further, 1000 observations for each of the $\operatorname{AR}(2)$ error disturbances with four pairs of autoregressive coefficients; (.2,-.9), (.8,-.9), (.2,-..7), and (.2,-..1) were also generated. Table (2) shows the values of autocorrelation coefficients $\rho_{1}, \rho_{2}$, disturbance variances, $\sigma_{\mathrm{u}}^{2}, \quad \sigma_{\mathrm{u}}^{2}=\left[\left(1-\phi_{2}^{2}\right)\left(1-\rho_{1}^{2}\right)\right]^{-1}$ and the relative efficiencies for estimating an intercept $\beta_{0}$, and the slope, $\beta_{1}$ of GLS to OLS for linear design with $\mathrm{T}=50$, denoted $\operatorname{RE}\left(\beta_{0}\right)$, and $\operatorname{RE}\left(\beta_{1}\right)$. Looking at the table, it may be seen that the choices $(.2,-.9)$ and $(.8,-.9)$ give the worst performance of OLS as compared to GLS for estimating $\left(\beta_{0}, \beta_{1}\right)$ of the regression coefficients and the largest values of $\sigma_{u}^{2}$. However, the choices $(.2,-.7)$ and $(.2,-.1)$ give the moderate and best performance of OLS as compared to GLS and the smallest values of $\sigma_{\mathrm{u}}^{2}$. Results for other sample sizes and designs demonstrate a similar pattern as in Table (2). 
The regression coefficients $\beta_{0}$, and $\beta_{1}$ for an intercept and the slope were each chosen to be equal one. Breusch (1980) has shown that for a fixed design, the distribution of $\frac{\hat{\beta}_{\mathrm{EGLS}}-\beta}{\sigma_{\mathrm{u}}^{2}}$ does not depend on the choice for $\beta$ and $\sigma_{u}^{2}$, and the result holds even if the covariance matrix $\Sigma$ is misspecified. When the design vector is stochastic, the assumption of a fixed design can be constructed as conditioning upon a given realization of the design, provided that the design is independent of $\mathrm{u}_{\mathrm{t}}$, Koreisha et al. (2002).

\section{Definition}

The efficiency of the GLS estimates relative to that of OLS in terms of the mean squared error of the regression coefficient, $\hat{\zeta}_{\beta_{j}}$, is given by:

$$
\hat{\zeta}_{\beta_{j}}=\frac{\sum_{\mathrm{i}=1}^{\mathrm{k}}\left(\widetilde{\beta}_{\mathrm{ij}, \mathrm{GLS}}-\beta_{\mathrm{j}}\right)^{2}}{\sum_{\mathrm{i}=1}^{\mathrm{k}}\left(\hat{\beta}_{\mathrm{ij}, \mathrm{OLS}}-\beta_{\mathrm{j}}\right)^{2}}
$$

where $\mathrm{j}=0,1$, for four GLS estimates, and $\mathrm{k}$ is the number of simulations. A ratio less than one indicates that the GLS estimates is more efficient than OLS, and if $\hat{\zeta}_{\beta_{j}}$ is close to one, then the OLS estimate is nearly as efficient as GLS estimates.

The Simulation Results for $\hat{\zeta}_{\beta_{j}}$

Tables (3) through (6) show the complete simulation results of the ratios of the GLS estimators relative to the OLS estimator in terms of the mean squared error of the regression coefficients, $\hat{\zeta}_{\beta_{0}}$ and $\hat{\zeta}_{\beta_{1}}$ in (7), when the serially correlated disturbance follows an $\mathrm{AR}(2)$ process. Each table presents the results for the three sample sizes considered, as well as all four selected pairs of AR(2) parametrizations. Each of the different designs is presented in a separate table.

Note that regardless of the sample size, selected design vectors, and $\operatorname{AR}(2)$ parametrizations the efficiency in estimating an intercept, $\beta_{0}$, and the slope, $\beta_{1}$, of the regression coefficients is higher for the GLS$\mathrm{AR}(2)$ estimator than OLS. This result emphasizes that GLS is the BLUE. However, OLS performs nearly as efficiently as GLS for all selected sample sizes and designs when $\Phi=$ $(.2,-.1)$. This result is not surprising since the choice of $\Phi=(.2,-.1)$ gives the highest performance of OLS as compared to GLS, in addition, it gives the smallest values of $\rho_{1}, \rho_{2}$, and $\sigma_{\mathrm{u}}^{2}$.

Table 2: Autocorrelation Coefficients, Disturbance Variances and the Relative Efficiencies of GLS to OLS for Standardized Linear Design with $\mathrm{T}=50$

\begin{tabular}{lccccc}
$\left(\phi_{1}, \phi_{2}\right)$ & $\rho_{1}$ & $\rho_{2}$ & $\sigma_{\mathrm{u}}^{2}$ & $\mathrm{RE}\left(\beta_{0}\right)$ & $\mathrm{RE}\left(\beta_{1}\right)$ \\
\hline$(.2,-.9)$ & .1053 & -.8789 & 5.3221 & .7656 & .5645 \\
$(.8,-.9)$ & .4211 & -.5632 & 6.3973 & .8325 & .6026 \\
$(.2,-.7)$ & .1176 & -.6765 & 1.9883 & .9414 & .8531 \\
$(.2,-.1)$ & .1818 & -.0636 & 1.0446 & .9993 & .9980
\end{tabular}


When the order of the disturbance term is under estimated, i.e. EIGLS-AR(1), the GLS estimate performs poorly. In fact, OLS is more efficient for nearly every situation considered here. For example, when $\Phi=(.8,-.9)$ for quadratic design with $\mathrm{T}=50,\left(\hat{\zeta}_{\beta_{0}}, \hat{\zeta}_{\beta_{1}}\right)=$ $(1.4179,1.7296)$ as shown in Table (3).

This shows that EIGLS-AR(1) can be much less efficient than OLS. The poor performance of EIGLS-AR(1) relative to OLS is most marked when the sample size is relatively estimation is smaller than an appropriate estimated AR structure. This suggests the small (i.e. $\mathrm{T}=50$ ) and the order of the autoregressive process used in the GLS surprising result that OLS may often be better than assuming an $\mathrm{AR}(1)$ when the actual process is $\operatorname{AR}(2)$. However, for the choice of $\Phi=(.2$, .1) there is little difference between OLS and EIGLS-AR(1). For example, for linear design with $\mathrm{T}=200, \quad\left(\hat{\zeta}_{\beta_{0}}, \hat{\zeta}_{\beta_{1}}\right)=(.9998, .9984)$ as presented in Table (4).

Table 3: Efficiency for MSEs of the Regression Coefficients of the GLS Estimators Relative to OLS Estimator for Quadratic Design

\begin{tabular}{|c|c|c|c|c|c|c|c|c|c|}
\hline \multirow[b]{3}{*}{ Size } & \multirow[b]{3}{*}{ Estimator } & \multicolumn{8}{|c|}{$\left(\Phi_{1}, \Phi_{2}\right)$} \\
\hline & & \multicolumn{2}{|c|}{$(.2,-.9)$} & \multicolumn{2}{|c|}{$(.8,-.9)$} & \multicolumn{2}{|c|}{$(.2,-.7)$} & \multicolumn{2}{|c|}{$(.2,-.1)$} \\
\hline & & $\hat{\zeta}_{\beta_{0}}$ & $\hat{\zeta}_{\beta_{1}}$ & $\hat{\zeta}_{\beta_{0}}$ & $\hat{\zeta}_{\beta_{1}}$ & $\hat{\zeta}_{\beta_{0}}$ & $\hat{\zeta}_{\beta_{1}}$ & $\hat{\zeta}_{\beta_{0}}$ & $\hat{\zeta}_{\beta_{1}}$ \\
\hline \multirow[t]{4}{*}{50} & GLS-AR(2) & 0.7929 & 0.5540 & 0.8321 & 0.6174 & 0.9435 & 0.8349 & 1.0002 & 0.9954 \\
\hline & EGLS-AR(2) & 0.7934 & 0.5567 & 0.8342 & 0.6172 & 0.9453 & 0.8409 & 1.0079 & 1.0094 \\
\hline & EIGLS-AR(1) & 1.0935 & 1.1973 & 1.4179 & 1.7296 & 1.0355 & 1.0861 & 1.0063 & 1.0050 \\
\hline & EIGLS-AR(4) & 0.7968 & 0.5623 & 0.8399 & 0.6182 & 0.9564 & 0.8500 & 1.0385 & 1.0332 \\
\hline \multirow[t]{4}{*}{100} & GLS-AR(2) & 0.8660 & 0.6950 & 0.8849 & 0.7104 & 0.9638 & 0.9287 & 1.0003 & 0.9993 \\
\hline & EGLS-AR(2) & 0.8661 & 0.6957 & 0.8844 & 0.7089 & 0.9676 & 0.9319 & 0.9993 & 0.9980 \\
\hline & EIGLS-AR(1) & 1.0453 & 1.0963 & 1.2136 & 1.4127 & 1.0207 & 1.0348 & 0.9989 & 1.0001 \\
\hline & EIGLS-AR(4) & 0.8651 & 0.6974 & 0.8861 & 0.7093 & 0.9723 & 0.9342 & 1.0078 & 1.0091 \\
\hline \multirow[t]{4}{*}{200} & GLS-AR(2) & 0.9410 & 0.8331 & 0.9700 & 0.8269 & 0.9628 & 0.9400 & 1.0004 & 0.9984 \\
\hline & EGLS-AR(2) & 0.9409 & 0.8326 & 0.9702 & 0.8265 & 0.9637 & 0.9400 & 1.0016 & 0.9990 \\
\hline & EIGLS-AR(1) & 1.0180 & 1.0417 & 1.0453 & 1.2683 & 1.0094 & 1.0187 & 1.0014 & 1.0023 \\
\hline & EIGLS-AR(4) & 0.9418 & 0.8338 & 0.9707 & 0.8290 & 0.9627 & 0.9407 & 1.0018 & 1.0021 \\
\hline
\end{tabular}


Table 4: Efficiency for MSEs of the Regression Coefficients of the GLS Estimators Relative to OLS Estimator for Linear Design

\begin{tabular}{|c|c|c|c|c|c|c|c|c|c|}
\hline \multirow[b]{2}{*}{ Size } & & \multicolumn{8}{|c|}{$\left(\Phi_{1}, \Phi_{2}\right)$} \\
\hline & \multirow[t]{2}{*}{ Estimator } & \multicolumn{2}{|c|}{$(.2,-.9)$} & \multicolumn{2}{|c|}{$(.8,-.9)$} & \multicolumn{2}{|c|}{$(.2,-.7)$} & \multicolumn{2}{|c|}{$(.2,-.1)$} \\
\hline & & $\hat{\zeta}_{\beta_{0}}$ & $\hat{\zeta}_{\beta_{1}}$ & $\hat{\zeta}_{\beta_{0}}$ & $\hat{\zeta}_{\beta_{1}}$ & $\hat{\zeta}_{\beta_{0}}$ & $\hat{\zeta}_{\beta_{1}}$ & $\hat{\zeta}_{\beta_{0}}$ & $\hat{\zeta}_{\beta_{1}}$ \\
\hline \multirow[t]{4}{*}{50} & GLS-AR(2) & 0.7472 & 0.5740 & 0.8214 & 0.6193 & 0.9740 & 0.8511 & 1.0012 & 0.9964 \\
\hline & EGLS-AR(2) & 0.7485 & 0.5771 & 0.8219 & 0.6200 & 0.9773 & 0.8548 & 1.0091 & 1.0073 \\
\hline & EIGLS-AR(1) & 1.1004 & 1.1893 & 1.3624 & 1.6995 & 1.0181 & 1.0895 & 1.0055 & 1.0079 \\
\hline & EIGLS-AR(4) & 0.7490 & 0.5824 & 0.8255 & 0.6220 & 0.9868 & 0.8595 & 1.0122 & 1.0448 \\
\hline \multirow[t]{4}{*}{100} & GLS-AR(2) & 0.8756 & 0.6641 & 0.8992 & 0.7340 & 0.9724 & 0.9204 & 1.0005 & 0.9996 \\
\hline & EGLS-AR(2) & 0.8766 & 0.6632 & 0.8992 & 0.7323 & 0.9718 & 0.9219 & 1.0025 & 1.0003 \\
\hline & EIGLS-AR(1) & 1.0349 & 1.0995 & 1.1826 & 1.4783 & 1.0156 & 1.0266 & 1.0025 & 1.0023 \\
\hline & EIGLS-AR(4) & 0.8782 & 0.6654 & 0.8992 & 0.7391 & 0.9758 & 0.9285 & 1.0133 & 1.0021 \\
\hline \multirow[t]{4}{*}{200} & GLS-AR(2) & 0.9127 & 0.8137 & 0.9584 & 0.8662 & 0.9623 & 0.9262 & 0.9990 & 0.9977 \\
\hline & EGLS-AR(2) & 0.9127 & 0.8135 & 0.9586 & 0.8662 & 0.9621 & 0.9271 & 1.0000 & 0.9980 \\
\hline & EIGLS-AR(1) & 1.0252 & 1.0464 & 1.0666 & 1.2104 & 1.0092 & 1.0175 & 0.9998 & 0.9984 \\
\hline & EIGLS-AR(4) & 0.9117 & 0.8123 & 0.9584 & 0.8668 & 0.9618 & 0.9255 & 1.0022 & 1.0032 \\
\hline
\end{tabular}

This result is expected because the choice of $\phi_{2}=-.1$ indicates that the serially correlated disturbance very nearly $\mathrm{AR}(1)$ since $\phi_{2}$ is close to zero.

To further demonstrate the efficiency of OLS, consider the quadratic and linear designs. OLS is nearly as efficient or more efficient in estimating $\left(\beta_{0}, \beta_{1}\right)$ than the GLS estimators; EGLS-AR(2), and EIGLS-AR(4), for moderate and large sample sizes (i.e. $\mathrm{T}=100$ and 200 ) with $\operatorname{AR}(2)$ parametrizations $\Phi=(.2,-.7)$ and $(.2$, .1) Tables (3) and (4). However, there are examples where OLS performs poorly as well. For the exponential design, OLS is nearly as efficient as EGLS-AR(2), and EIGLS-AR(4) for all sample sizes only when $\Phi=(.2,-.1)$.
Otherwise, OLS performs poorly as shown in Table (5). For example, when $\mathrm{T}=50$ with $\Phi=$ $(.2,-.9), \hat{\zeta}_{\beta_{1}}=(.2035, .2108)$. However, even in this case, the performance of the OLS estimator for estimating the intercept is not bad, $\hat{\zeta}_{\beta_{0}}=$ (.7561, .7606). In fact, the performance of OLS is always better for estimating the intercept than the slope.

For the standard Normal stochastic design model, OLS fares more poorly. Only for $\Phi=(.2,-.1)$ does the efficiency of OLS match GLS as shown in Table (6). However, regardless of the sample size, OLS performs as nearly as efficiently or better than EIGLS-AR(1) for all selected autoregressive coefficients for estimating $\beta_{0}$. 
Table 5: Efficiency for MSEs of the Regression Coefficients of the GLS Estimators Relative to OLS Estimator for Exponential Design

\begin{tabular}{|c|c|c|c|c|c|c|c|c|c|}
\hline \multirow[b]{3}{*}{ Size } & \multirow[b]{3}{*}{ Estimator } & \multicolumn{8}{|c|}{$\left(\Phi_{1}, \Phi_{2}\right)$} \\
\hline & & \multicolumn{2}{|c|}{$(.2,-.9)$} & \multicolumn{2}{|c|}{$(.8,-.9)$} & \multicolumn{2}{|c|}{$(.2,-.7)$} & \multicolumn{2}{|c|}{$(.2,-.1)$} \\
\hline & & $\hat{\zeta}_{\beta_{0}}$ & $\hat{\zeta}_{\beta_{1}}$ & $\hat{\zeta}_{\beta_{0}}$ & $\hat{\zeta}_{\beta_{1}}$ & $\hat{\zeta}_{\beta_{0}}$ & $\hat{\zeta}_{\beta_{1}}$ & $\hat{\zeta}_{\beta_{0}}$ & $\hat{\zeta}_{\beta_{1}}$ \\
\hline \multirow[t]{4}{*}{50} & GLS-AR(2) & 0.7529 & 0.1951 & 0.8208 & 0.2160 & 0.9394 & 0.5576 & 0.9986 & 0.9706 \\
\hline & EGLS-AR(2) & 0.7561 & 0.2035 & 0.8256 & 0.2241 & 0.9464 & 0.5642 & 0.9987 & 1.0030 \\
\hline & EIGLS-AR(1) & 1.0451 & 1.1030 & 1.1649 & 1.1683 & 1.0167 & 1.0656 & 0.9969 & 1.0019 \\
\hline & EIGLS-AR(4) & 0.7606 & 0.2108 & 0.8293 & 0.2322 & 0.9473 & 0.5815 & 1.0042 & 1.0775 \\
\hline \multirow[t]{4}{*}{100} & GLS-AR(2) & 0.8922 & 0.1979 & 0.9139 & 0.2311 & 0.9668 & 0.5461 & 1.0009 & 0.9830 \\
\hline & EGLS-AR(2) & 0.8895 & 0.2021 & 0.9163 & 0.2353 & 0.9682 & 0.5467 & 0.9993 & 0.9980 \\
\hline & EIGLS-AR(1) & 1.0115 & 1.0803 & 1.0893 & 1.1383 & 1.0077 & 1.0575 & 0.9991 & 0.9965 \\
\hline & EIGLS-AR(4) & 0.8904 & 0.2068 & 0.9149 & 0.2357 & 0.9692 & 0.5578 & 0.9997 & 1.0187 \\
\hline \multirow[t]{4}{*}{200} & GLS-AR(2) & 0.9168 & 0.2139 & 0.9771 & 0.2084 & 1.0162 & 0.5293 & 1.0022 & 0.9877 \\
\hline & EGLS-AR(2) & 0.9164 & 0.2143 & 0.9782 & 0.2132 & 1.0150 & 0.5303 & 1.0008 & 0.9990 \\
\hline & EIGLS-AR(1) & 1.0053 & 1.0645 & 1.0492 & 1.2352 & 0.9999 & 1.0390 & 1.0012 & 0.9900 \\
\hline & EIGLS-AR(4) & 0.9171 & 0.2161 & 0.9802 & 0.2151 & 1.0149 & 0.5425 & 1.0006 & 1.0062 \\
\hline
\end{tabular}


Table 6: Efficiency for MSEs of the Regression Coefficients of the GLS Estimators Relative to OLS Estimator Standard Normal Stochastic Design

\begin{tabular}{|c|c|c|c|c|c|c|c|c|c|}
\hline \multirow[b]{3}{*}{ Size } & & \multicolumn{8}{|c|}{$\left(\Phi_{1}, \Phi_{2}\right)$} \\
\hline & & \multicolumn{2}{|c|}{$(.2,-.9)$} & \multicolumn{2}{|c|}{$(.8,-.9)$} & \multicolumn{2}{|c|}{$(.2,-.7)$} & \multicolumn{2}{|c|}{$(.2,-.1)$} \\
\hline & Estimator & $\hat{\zeta}_{\beta_{0}}$ & $\hat{\zeta}_{\beta_{1}}$ & $\hat{\zeta}_{\beta_{0}}$ & $\hat{\zeta}_{\beta_{1}}$ & $\hat{\zeta}_{\beta_{0}}$ & $\hat{\zeta}_{\beta_{1}}$ & $\hat{\zeta}_{\beta_{0}}$ & $\hat{\zeta}_{\beta_{1}}$ \\
\hline \multirow[t]{4}{*}{50} & GLS-AR(2) & 0.6416 & 0.1127 & 0.7427 & 0.0667 & 0.8851 & 0.3334 & 0.9998 & 0.8838 \\
\hline & EGLS-AR(2) & 0.6437 & 0.1149 & 0.7442 & 0.0676 & 0.8807 & 0.3428 & 1.0176 & 0.9513 \\
\hline & EIGLS-AR(1) & 1.0536 & 0.9591 & 1.2168 & 0.5186 & 1.0129 & 0.9598 & 1.0091 & 0.9244 \\
\hline & EIGLS-AR(4) & 0.6509 & 0.1211 & 0.7477 & 0.0737 & 0.8908 & 0.3652 & 1.0385 & 1.0221 \\
\hline \multirow[t]{4}{*}{100} & GLS-AR(2) & 0.7601 & 0.1055 & 0.8466 & 0.0640 & 0.9350 & 0.3230 & 0.9978 & 0.8902 \\
\hline & EGLS-AR(2) & 0.7598 & 0.1060 & 0.8472 & 0.0639 & 0.9341 & 0.3274 & 0.9984 & 0.9076 \\
\hline & EIGLS-AR(1) & 1.0241 & 0.9568 & 1.1141 & 0.5261 & 1.0121 & 0.9546 & 0.9994 & 0.9158 \\
\hline & EIGLS-AR(4) & 0.7611 & 0.1109 & 0.8477 & 0.0668 & 0.9346 & 0.3386 & 1.0033 & 0.9359 \\
\hline \multirow[t]{4}{*}{200} & GLS-AR(2) & 0.8624 & 0.1002 & 0.9323 & 0.0720 & 0.9715 & 0.3226 & 1.0038 & 0.9194 \\
\hline & EGLS-AR(2) & 0.8628 & 0.1006 & 0.9319 & 0.0725 & 0.9707 & 0.3245 & 1.0028 & 0.9331 \\
\hline & EIGLS-AR(1) & 1.0141 & 0.9581 & 1.0400 & 0.5181 & 1.0021 & 0.9598 & 1.0033 & 0.9418 \\
\hline & EIGLS-AR(4) & 0.8630 & 0.1033 & 0.9314 & 0.0748 & 0.9719 & 0.3307 & 1.0058 & 0.9512 \\
\hline
\end{tabular}

\section{Discussion}

In investigating the simulation results in the previous section, the following significant results were observed. First and foremost, it was noticed that regardless of the sample size for all design structures and selected autoregressive coefficients, the efficiency in estimating an intercept, $\beta_{0}$, and the slope, $\beta_{1}$, of the regression model is higher for the GLS estimator based on the correct disturbance model structures and known $\operatorname{AR}(2)$ coefficients. This result is expected since GLS is BLUE, but because GLS requires a priori knowledge of $\Sigma$, this is not a viable option.
In addition, the relative efficiency of OLS is better than EIGLS-AR(1) in estimating $\left(\beta_{0}, \beta_{1}\right)$ for all sample sizes and nonstochastic design vectors. The relative efficiency of OLS to be superior to that of EIGLS in estimating the slope when $\mathrm{T}=50$ with $\mathrm{AR}(2)$ parametrization $(.8,-.9)$ was also observed. This choice of $(.8$, .9) gives the highest first-order autoregressive coefficient $\left(\rho_{1}=.4211\right)$ and largest variance of the error process $\left(\sigma_{u}^{2}=6.3973\right)$ among the other choices of $\operatorname{AR}(2)$ parametrizations. This explains the poor relative performance of OLS to GLS for this choice of parameter. 
However, from Table (3) through Table (6), it may be seen that the performance of EIGLS$\mathrm{AR}(1)$ is even worse. This appears to occur because $\operatorname{AR}(2)$ parametrization $(.8,-.9)$ produces large values of $\rho_{1}, \rho_{2}$ in absolute value $\left(\rho_{2}=-.5632\right)$ and disturbance variance comparing to the other parameter choices. This means using OLS is better than assuming another incorrect error process.

The third general conclusion from the simulation study is that regardless of the sample size, all of the estimators perform equally well with $\operatorname{AR}(2)$ parametrization $\quad(.2,-.1)$. This result is not surprising because the choice of $(.2$, -.1) gives the smallest variance of the process $\left(\sigma_{u}^{2}=1.0446\right)$, which is sufficiently close to the variance of standard OLS.

Fourth, for all stochastic and nonstochastic design vectors, the differences in the relative efficiency of OLS and all GLS estimators in estimating $\beta_{0}$ with a few expected exceptions are negligible. In fact, this is so even when the variance of the process is large, in other words, when $\operatorname{AR}(2)$ parametrizations are $(.2,-.9)$ and $(.8,-.9)$.

Similar to results for section 2, when the design vector is linear or quadratic, the relative efficiency of OLS is nearly as good as the EGLS-AR(2) and EIGLS-AR(4) estimators for moderate and large sample sizes for estimating $\beta_{1}$ with small variance of the disturbances.

It is observed that the differences in the relative efficiencies of GLS-AR(2), EGLS$\operatorname{AR}(2)$, and EIGLS-AR(4) in estimating $\left(\beta_{0}, \beta_{1}\right)$ are insignificant. Hence, when confronted with an error with unknown order $p$, it appears that using $\mathrm{AR}(4)$ is the best bet.

Finally, OLS may often be more preferable than assuming an $\mathrm{AR}(1)$ process when the actual process is $\operatorname{AR}(2)$. In other words, it is sometimes better to ignore the autocorrelation of the disturbance term and use the OLS estimation rather than to incorrectly assume the process is an $\mathrm{AR}(1)$.

\section{Future Research \\ Perhaps, even more important than the efficiency of the different estimation methods in}

these models, is the effect on forecasting performance. Koreisha et al. (2004) investigated the impact that EIGLS correction may have on forecast performance. They developed a new procedure for generating forecasts for regression models with auto-correlated disturbances based on OLS and a finite AR process. They found that for predictive purposes there is not much gained in trying to identify the actual order and form of the auto-correlated disturbances or using more complicated estimation methods such as GLS or MLE procedures, which often require inversion of large matrices. It is necessary to extend Koreisha et al. (2004) results for different design vectors of the independent variables including both stochastic and nonstochastic designs instead of using one independent variable generated by an $\mathrm{AR}(1)$ process as in their investigation.

A second important consideration is the estimation of the standard errors of the estimators. In practice, if one were using a statistical package to compute the OLS estimators the variance estimate produced would be based on $\sigma_{u}^{2}\left(X^{\prime} X\right)^{-1}$, which may be biased for the true variance $\sigma_{\mathrm{u}}^{2}\left(\mathrm{X}^{\prime} \mathrm{X}\right)^{-1} \mathrm{X}^{\prime} \sum \mathrm{X}\left(\mathrm{X}^{\prime} \mathrm{X}\right)^{-1}$. For GLS estimation ( $\Sigma$ known), on the other hand, the variance estimate is unbiased for the true variance of the GLS estimator. It is unclear, however, how the variance estimators for EGLS estimation behave. The impact that the variance estimators may have on inference based on the OLS estimator is currently being investigated.

Finally, the long range goal is the creation of guidelines or rules of thumb which will aid the practitioner when deciding which regression estimation procedure to use.

\section{Conclusion}

This article has investigated an important statistical problem concerning estimation of the regression coefficients in the presence of autocorrelated disturbances. In particular, the comparison of efficiency of the ordinary least squares (OLS) estimation to alternative procedures such as generalized least squares (GLS) and estimated GLS (EGLS) estimators in the presence of autocorrelated disturbances was 
discussed. Both stochastic and non-stochastic design vectors were used with different sample sizes.

It was found that regardless of the sample size, design vector, and order of the auto-correlated disturbances, the relative efficiency of the OLS estimator generally increases with decreasing values of the disturbance variances. In particular, if the disturbance structure is a first or second order autoregressive and the dependent variable is nonstochastic and linear or quadratic, OLS performs nearly as well as its competitors for small values of the disturbance variances. The gain in efficiency of the GLS estimator for different design vectors such as exponential and standard Normal compared to the OLS estimator is substantial for moderate and large values of the autoregressive coefficient in the case of an $\mathrm{AR}(1)$ process and large values of the disturbance variance in the presence of an $\operatorname{AR}(2)$ process. However, for small values of the autoregressive coefficient and disturbance variance the OLS estimator appears to be nearly as efficient as the GLS estimator.

It was also found that if the error structure is autoregressive, and the dependent variable is nonstochastic and linear or quadratic, the OLS estimator performs nearly as well as its competitors. When faced with an unknown error structure, however, AR(4) may be the best choice.

\section{References}

Anderson, T. W. (1948). On the theory of testing serial correlation. Skandinavisk Aktuarietid skrift, 31, 88-116.

Anderson, T. W. (1971). The Statistical analysis of time series. New York:, N.Y: Wiley.

Breusch, T. (1980). Useful invariance results for generalized regression models. Journal of Econometrics, 13, 327-340.
Chipman, J. S. (1979). Efficiency of least squares estimation of linear trend when residuals are autocorrelated. Econometrica, 47, 115-128.

Choudhury, A., Hubata, R. \& Louis, R. (1999). Understanding time-series regression estimators. The American Statistician, 53, 342348.

Grenander, U. \& Rosenblatt, M. (1957). Statistical analysis of stationary time series. New York, N.Y.: Wiley.

Judge, G. G., Griffiths, W. E., Hill, R. C.. Lutkepohl, H., \& Lee, T. C. (1985). The theory and practice of econometrics. New York, N.Y.: Wiley \& Sons Inc.

Kadiyala, K. R. (1968). A transformation used to circumvent the problem of autocorrelation. Econometrica, 36, 93-96.

Koreisha, S. G. and Fang, Y. (2002). Generalized least squares with misspecified serial correlation structures. Journal of the Royal Statistical Society, 63, Series B, 515-531.

Koreisha, S. G. and Fang, Y. (2004). Forecasting with serially correlated regression models. Journal of Statistical Computations and Simulation, 74, 625-649.

Kramer, W. (1980). Finite sample efficiency of ordinary least squares in the linear regression model with autocorrelated errors. Journal of the American Statistical Association, 75, 1005-1009.

Maeshiro, A. (1976). Autoregressive transformation, trended independent variables and autocorrelated disturbances terms. Review of Economics and Statistics, 58, 497-500.

Maeshiro, A. (1979). On the retention of the first observations in serial correlation adjustment of regression models. International Economic Review, 20, 259-65.

Ullah, A., Srivastava, V. K., Magee, L., \& Srivastava, A. (1983). Estimation of linear regression model with autocorrelated disturbances. Journal of Time Series Analysis, 4, 127-135. 